\title{
Is there a link between long-term statin use and reduced gallstone risk?
}

\section{"...decreased cholesterol biosynthesis may lead to decreased hepatic cholesterol secretion and consequently to increased bile solubility, which may lower the risk of gallstone formation."}

We recently published the results of an observational study in which we analyzed a large number of patients who underwent cholecystectomy. Patients who regularly used statins for approximately 1 year or more had a substantially reduced risk of developing a first-time diagnosis of gallstones followed by cholecystectomy compared with patients not using statins [1]. Does this finding reflect an important association of considerable clinical and public health relevance?

Statins decrease the de novo biosynthesis of cholesterol in the liver in a dose-dependent manner by inhibiting the enzyme 3-hydroxy3-methyl-glutaryl-coenzyme A reductase - the rate limiting step in hepatic cholesterol biosynthesis. Consequently, the synthesis of LDL-receptors on the surface of hepatocytes is increased and the serum level of LDL-C decreases significantly owing to increased hepatic clearance of LDL from the blood. Statins also reduce LDL serum levels by enhancing removal of VLDL and intermediate density lipoprotein, and by decreasing hepatic VLDL production. Furthermore, statins usually decrease plasma triglyceride levels and slightly increase HDL-C levels. Usually, maximal effects on serum cholesterol levels are achieved within a few weeks.

Gallstones are an important cause of morbidity in the Western world, leading to considerable morbidity and not rarely requiring surgical cholecystectomy. The majority ( $\sim 0 \%)$ of gallstones in the Western world are cholesterol stones; the remaining are black and brown pigment stones. Known important risk factors for cholesterol stones are obesity, female sex, increasing age and postmenopausal estrogen-replacement therapy. Potential protective factors are regular physical activity, high fruit and vegetable diet and coffee consumption. While decreased HDL and increased triglyceride levels have been linked to an increased risk of gallstone formation in most epidemiological and clinical studies, there is conflicting evidence on whether altered LDL levels are associated with an altered gallstone risk [2]. Several prospective studies reported no association between LDL levels and gallstone risk, whereas other authors reported either a decreased or an increased risk of gallstone disease in relation to increased LDL levels. Reasons for these inconsistencies encompass differences in study design, study population, measurement method, bias or residual confounding in population-based studies.

\section{"Gallstones are an important cause of morbidity in the Western world, leading to considerable morbidity and not rarely requiring surgical cholecystectomy."}

It is widely recognized that cholesterol hypersecretion is crucial in gallstone formation; it can be accompanied by high or low secretion rates of biliary acids and phospholipid. In addition, cholesterol supersaturation, accelerated nucleation and crystallization and gallbladder hypomotility have also been demonstrated to contribute to gallstone formation. To date, various animal studies and small clinical studies in humans have been performed, revealing conflicting results as to whether the use of statins may decrease bile lithogenicity [3-9]. In theory, decreased cholesterol biosynthesis may lead to decreased hepatic cholesterol secretion and consequently to increased bile solubility, which may lower the risk of gallstone formation. Several, but not all, studies demonstrated decreased cholesterol secretion into bile during statin treatment. Furthermore, higher serum levels of HDL due to statin use may also be beneficial, since decreased HDL serum levels have been linked to an increased risk of gallstone disease. On the other hand, decreased cholesterol biosynthesis

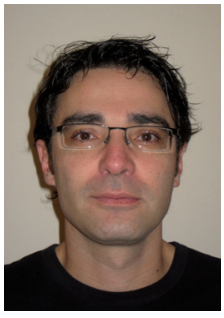

Michael Bodmer

Division of Clinical

Pharmacology \& Toxicology,

University Hospital Basel,

Switzerland

and

Rocky Mountain Poison \& Drug

Center, Denver Health, Denver,

CO, USA

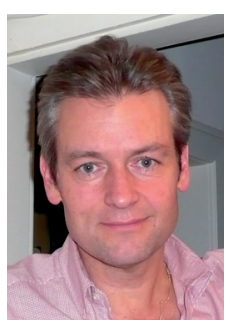

\section{Christoph R Meier}

Author for correspondence:

Basel Pharmacoepidemiology

Unit, Hospital Pharmacy,

University Hospital Basel,

Spitalstrasse 26,

$\mathrm{CH}-403$ I Basel,

Switzerland

Tel.: +4I 615565369

Fax: +4I 612658875

meierch@uhbs.ch

and

Boston Collaborative Drug

Surveillance Program, Boston

University School of Medicine,

MA, USA

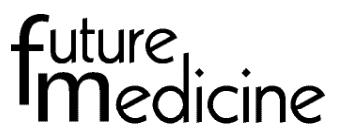


may be associated with diminished formation and secretion of hydrophilic bile acids, possibly resulting in decreased bile solubility. However, the majority of studies reported unchanged bile acid synthesis in humans using statins.

\section{“...short-term use of statins was not associated with a decreased risk of gallbladder disease followed by cholecystectomy."}

The question arises as to whether the risk of developing clinically manifest gallstone disease is altered by statin use or not. A possible association between statin use and an altered gallstone risk has been explored in three large observational studies so far $[1,10,11]$. In 2001, Simon et al. investigated whether estrogen and progestin supplementation exhibited an increased risk for bile tract surgery (mainly cholecystectomy owing to gallstone disease) in a randomized, placebocontrolled trial [11]. Even though use of statins was not the major focus of the study, the authors noticed that statins were independently associated with a reduced risk for bile tract surgery. However, the authors did not provide information on duration or dosage of statin use. In a large cohort encompassing more than 50,000 women participating in the Nurses' Health Study, Tsai and coworkers recently analyzed the risk of selfreported gallstone disease and cholecystectomy in association with the use of statins [12]. The authors found a moderately decreased adjusted relative risk estimate of cholecystectomy for current statin users of 0.82 (95\% CI: 0.70-0.96) compared with nonusers. Again, the investigators did not report details on exposure time or dose, and the study was restricted to women. The third study exploring the effect of statin use on the risk of gallstone disease followed by cholecystectomy was carried out in 2009 by our group, the Basel Pharmacoepidemiology Unit at the University Hospital of Basel, Switzerland [1]. We used the UK-based General Practice Research Database (GPRD) to conduct a large case-control analysis, encompassing 27,035 cases with gallstone disease followed by cholecystectomy and 106,531 controls, matched to cases for age, sex, calendar time and general practice attended. We found a substantially decreased odds ratio for cholecystectomy for long-term users ( $>1-1.5$ years) of statins of 0.64 (95\% CI: 0.59-0.70) compared with nonusers. Notably, short-term use of statins was not associated with a decreased risk of gallbladder disease followed by cholecystectomy. These results were observed across all different statins, and there was a tendency towards a lower relative risk estimate in patients using higher statin doses. However, in contrast to Simon et al., we were not able to relate the findings to serum cholesterol levels or history of life style habits. However, in their study, LDL, HDL and triglyceride serum levels were not independently associated with an altered risk of biliary tract surgery [12]. Although it is recognized that, among other factors, high saturated and trans-fat intake, low fiber intake and low physical activity are associated with an increased risk of gallstones [2,13], all of these parameters seemed not to be independent risk factors of cholecystectomy in the study by Tsai and coworkers [12]. Furthermore, statin users tended to have other, more relevant risk factors for gallstone disease compared with nonusers, such as heavier weight, less physical activity, less coffee consumption or use of hormone replacements, which makes the finding related to statins even more intriguing [12].

\section{"...statin users tended to have other, more relevant risk factors for gallstone disease compared with nonusers..."}

Taken together, these three studies, all based on different study designs and including thousands of patients, strengthen the hypothesis that statin use may decrease the risk of gallstone disease followed by cholecystectomy in clinical practice. However, it must be noted that observational data do not allow causality to be proven; such studies can only provide evidence for a possible association. Therefore, a possible causal association needs to be confirmed in a randomized prospective trial. However, observational studies are the only way to explore such a hypothesis, since animal models or observations in single patients do not justify the conduction of a prospective randomized trial. In such a case, observational studies using data from established databases of high quality can be helpful in exploring whether there is any evidence that an alleged association of interest may be of clinical relevance at all or not. In this case, current evidence from observational research does provide intriguing evidence that statins may indeed reduce the risk of gallstone disease to a considerable degree.

Several questions remain to be answered. First, despite a substantial amount of available data in the literature, no definite mechanism of action on how statins may alter bile lithogenicity 
has been established to date. Second, the time dependence of the observed risk reduction was assessed in only one study in which the risk reduction became evident only after longer-term statin use of 1 year or more. By contrast, to our knowledge, the previous clinical trials investigating a possible effect of statins on bile lithogenicity that reported such an effect did so after short-term use [3-6]. Third, bringing together all available evidence from the literature, high levels of LDL per se (i.e., the most important indication for statins) are unlikely to be independently linked to an increased risk of gallstone formation. Therefore, decreased serum levels of LDL do not seem to be directly related to a potentially decreased risk of gallstone disease in statin users. Fourth, to date, no prospective randomized trial has been reported assessing the risk of gallstone disease followed by cholecystectomy in users of statins compared with nonusers. Given the substantial morbidity and the high economic burden of gallstone disease in the Western world, the results of such a trial would be highly welcome. To date, despite intriguing findings in observational studies, there is not sufficient evidence to recommend statin therapy to lower the risk for gallstone disease. However, a possible risk reduction of gallstone disease may be looked at as a desired side-effect of statins, if the findings can be confirmed.

Financial \& competing interests disclosure

Christoph R Meier has consulted or is consulting for companies producing and selling statins, in particular AstraZeneca (DE, USA) and Novartis (Switzerland). The authors have no other relevant affiliations or financial involvement with any organization or entity with a financial interest in or financial conflict with the subject matter or materials discussed in the manuscript apart from those disclosed.

No writing assistance was utilized in the production of this manuscript.

\section{Bibliography}

1 Bodmer M, Brauchli YB, Krahenbuhl S, Jick SS, Meier CR: Statin use and risk of gallstone disease followed by cholecystectomy. JAMA 302 (18), 2001-2007 (2009).

2 Shaffer EA: Gallstone disease: epidemiology of gallbladder stone disease. Best Pract. Res. Clin. Gastroenterol. 20(6), 981-996 (2006).

3 Chapman BA, Burt MJ, Chisholm RJ, Allan RB, Yeo KH, Ross AG: Dissolution of gallstones iwth simvastatin, an HMG CoA reductase inhibitor. Dig. Dis. Sci. 43(2), 349-353 (1998).

4 Porsch-Ozcürümez M, Hardt PD, Schnell-Kretschmer $\mathrm{H}$ et al.: Effect of fluvustatin on binary lipids in subjects with an elevated cholesterol saturation index. Eur. J. Clin. Pharmacol. 56(12), 873-978 (2001).

5 Smith JL, Roach PD, Wittenberg LN et al.: Effects of simvastatin on hepatic cholesterol metabolism, bile lithogenicity and bile acid hydrophobicity in patients with gallstones. J. Gastroenterol. Hepatol. 15(8), 871-879 (2001).
6 Wilson IR, Hurrell MA, Pattinson NR, Chapman BA: The effect of simvastatin and bezafibrate on bile composition and gall-bladder emptying in female non-insulindependent diabetics. J. Gastroenterol. Hepatol. 9(5), 447-451 (1994).

7 Miettinen TE, Kiviluoto T, Taavitsainen M, Vuoristo M, Miettinen TA: Cholesterol metabolism and serum and biliary noncholesterol sterols in gallstone patients during simvastatin and ursodeoxycholic acid treatments. Hepatology 27(3), 649-655 (1998).

Sharma BC, Agarwal DK, Baijal SS, Saraswat VA: Pravastatin has no effect on bile lipid composition, nucleation time, and gallbladder motility in persons with normal levels of cholesterol. J. Clin. Gastroenterol. 25(2), 433-436 (1997).

9 Smit JW, van Erpecum KJ, Renooij W et al.: The effects of the 3-hydroxy, 3-methylglutaryl coenzyme A reductase inhibitor pravastatin on bile composition and nucleation of cholesterol crystals in cholesterol gallstone disease. Hepatology 21(6), 1523-1529 (1995).
10 Tsai CJ, Leitzmann MF, Willett WC, Giovannucci EL: Statin use and the risk of cholecystectomy in women. Gastroenterology 136(5), 1593-1600 (2009).

11 Simon JA, Hunninghake DB, Agarwal SK et al: : Effect of estrogen plus progestin on risk for biliary tract surgery in postmenopausal women with coronary artery disease. The Heart and Estrogen/Progestin Replacement Study. Ann. Intern. Med. 135(7), 493-501 (2001).

12 Tsai CJ, Leitzmann MF, Willett WC, Giovannucci EL: Statin use and the risk of cholecystectomy in women. Gastroenterology 136(5), 1593-1600 (2009).

13 Tsai CJ, Leitzmann MF, Willett WC, Giovannucci EL: Long-chain saturated fatty acids consumption and risk of gallstone disease among men. Ann. Surg. 247(1), 95-103 (2008). 